\title{
13 Training Beginners: Bird Measurements
}

Measurements are of value to science when they are reproducible, i.e. do not depend on the individual characteristics of the person. The compatibility of measurements does not imply that two people present identical results for each bird measured independently by them, but rather, it implies statistical concordance for a series of measurements done by a number of people measuring the same sample of birds. This situation is attainable when the standard techniques are carried out strictly according to the rules described. The system of instruction must guarantee correct interpretation of these standard descriptions of techniques, ensure correct execution of measurements and cross check the results of this instruction.

Measurers are trained step by step as follows:

1. The measurer reads the descriptions of the standard technique, and the instructor explains them in order to cover any doubts that might ensue.

2. The instructor demonstrates the correct way of making measurements (slowly, with comments) and provides specimens showing typical fat deposit patterns. This step is complete on a small number of birds.

3. The measurer practises their fresh knowledge on a few specimens, under the guidance of the instructor. The goal is to attain measurements matching those of the instructor, who has measured the bird beforehand. Measure about 20 birds in this phase.

4. The measurer independently measures birds previously measured by the instructor (about 100 birds), then compares these results to those already noted and corrects their errors. When more regular errors are perceived, the measurer should discuss shortcomings with the instructor.

5. Initial checking:

a. The beginner measures a series of about 50 specimens independently and without recourse to comparative measurements. His results are noted separately on a training chart. This series should involve birds of comparable size. The optimal, full control involves birds of the Goldcrest, Great Tit and Thrush size in order to detect possible size-dependent errors in measurements;

b. After completion of the series, the beginner's results are compared with the correct measurements and all deviations are noted using coloured numbers at the corner of every line on the chart where results differ. These deviations are summarised algebraically for every parameter separately and the mean deviations are calculated. Deviations of less than 0.2 are treated as a correct result;

c. If a greater mean deviation occurs, the instructor should carefully check the probable reasons for incorrect measurements, repeat stages 3 and 4 of the learning process and superintend until reproducible results have been achieved. 


\section{Final check:}

Following a positive initial check, the beginner should measure some hundred birds alone and the instructor's checking, as under point 5, is repeated. It is worthwhile to check the beginner in the following season when his performance should have stabilized, or individual divergences will have emerged. The check is best performed if all the beginners of the research group can be compared with the "group-standard" or even the "country-standard" person.

Later, during work, there is a good custom to make comparisons between ringers when changes of measuring person occur: both persons should double measure some individuals or measure some, but more, individuals from the same group of migrants. The other solution, if there is no possibility to stay at the ringing station for a few days, is to compare measurements ex post on graphs made from the data collected in subsequent days as it was shown at the original graphs. In this case, use data about the most common bird is recommended. 